\title{
2. SOME REMARKS ON MULTIPLE GALAXIES
}

\author{
V. A. AMBARTSUMIAN \\ Bjurakan Observatory, Erevan, U.S.S.R.
}

(Read by Mrs Masevich)

Within each cluster of galaxies, the ratio of the number of multiple galaxies to the number of single galaxies is much higher than the ratio calculated under the assumption of statistical equilibrium. This fact leads, without any supplementary assumption, to the conclusion that the components of any given multiple galaxy have a common origin. In this respect the multiple galaxies are similar to the multiple stars of our stellar system.

The study of the configurations of the multiple galaxies in Holmberg's catalogue has shown that among 132 multiple systems in this catalogue, 87 systems $(65 \%)$ form configurations of the Trapezium type, while only $27(20 \%)$ belong to the type usual for multiple star systems. The remaining I $5 \%$ are intermediate in type with the ratios of the largest distances between components to the smallest ones between $2 \cdot 5$ and $3 \cdot 0$.

In this respect the class of multiple galaxies differs strongly from the class of multiple stars, in which the Trapezium type configurations form only a small minority.

The Trapezium type multiple galaxies are probably unstable, at least in the cases in which the masses of the components are similar. The very existence of such configurations makes it probable that many of them have positive total energies. This means that some of the multiple galaxies represent newly formed and expanding groups, but this assumption requires some independent proof.

From the differences in the radial velocities, $\Delta v_{r}$, between the components of the pairs of galaxies observed by Page and others, we can find the values of $\rho\left(\Delta v_{r}\right)^{2}$, where $\rho$ is the projected linear distance between the components. These values of $\rho\left(\Delta v_{r}\right)^{2}$ are systematically much higher for the pairs in the multiple systems with three or more components than in simple double galaxies.

However, if all the systems have negative total energies, the average value of $\rho\left(\Delta v_{r}\right)^{2}$ will be proportional to the average mass of the systems. Thus, 
the assumption of negative total energy for all systems leads to the conclusion that the masses of the components of multiple galaxies are systematically greater (at least by multiples of two or three) than the masses of the components of double galaxies.

The only way to avoid this conclusion is to assume that some multiple galaxies have positive energies. Thus we have an indirect proof of the assumption introduced above.

The mean value of the ratio $f=M / L$, derived for multiple systems, assuming negative total energies, is much higher than the maximum value of the same ratio obtained from the rotation of individual galaxies. This may provide additional evidence in favour of positive total energies for some multiple galaxies.

If we assume the steady state of clusters of galaxies and apply the virial theorem, we obtain very high values for the ratio $f$. Thus we obtain $f=2000$ for the Virgo cluster and $f=5000$ for the Coma cluster, if we take the new value determined by Zwicky for the radius of the latter cluster (and not $f=800$ as derived by Schwarzschild, who applied only the corrections which tend to minimize the mass and neglected the opposite corrections).

Though the evidence in favour of inter-galactic matter in clusters of galaxies is convincing, it seems improbable that the mass of this matter in a cluster can exceed many times the total mass of member galaxies. Instead, it seems that the nature of inter-galactic patches is similar to that of irregular galaxies in which the ratio $f$ is very small. Therefore it is reasonable to assume that some of the clusters of galaxies are systems of positive energy. In this case the virial theorem is not applicable.

As Markarian has pointed out, the presence of a chain of bright galaxies in the dense part of the Virgo cluster is an evidence in favour of the nonsteady state of this system. The chain includes the giant elliptic galaxies M 84 and M 86 and is one of the outstanding features of the cluster. In this respect there is a remarkable similarity between the irregular clusters of galaxies and the O-associations, in which chains of blue super-giants appear (for example, the Orion Belt).

If among the multiple galaxies we have systems of positive energy, it is also natural to expect the occurrence of such cases among double galaxies. A double galaxy of this type would represent a pair of mutally receding stellar systems. There is no configuration-criterion in the case of double systems. However, the question arises of the possibility of observing such pairs in the early stages when the mutual distance of the components is very small and the nucleus of one component is situated in the central part of the other. 
Actually, we observe some such narrow pairs as radio-galaxies. It is easy to show, applying statistical considerations, that the galaxy NGC 5128 (Centaurus A) cannot be a result of the collisions of two previously independent galaxies. In the case of Cygnus A the collision hypothesis should be abandoned on the grounds of similar statistical reasoning. In the case of NGC 4486 (Virgo A) the division of the nucleus into the main mass and a jet was directly observed by Baade. Therefore, we may consider a radio galaxy as a result of the division of the nucleus of some primary system.

\section{Discussion}

Minkowski: Results of a detailed investigation of NGC 1275, reported at the Jodrell Bank Symposium, admit no other interpretation than that this system consists of two colliding galaxies. 